\title{
Dried tube specimen preparation and stability validation for brucellosis serological external quality assessment and quality control materials in resource-limited settings
}

\author{
Arsen Zakaryan ${ }^{1}$, Julius Manjengwa ${ }^{1}$, Hrant Danielyan², Pertsh Tumanyan ${ }^{3}$, Zaruhi \\ Davtyan $^{1}$, Obert Kachuwaire ${ }^{1}$, and Antoine Pierson ${ }^{1}$ \\ ${ }^{1}$ Integrated Quality Laboratory Services \\ ${ }^{2}$ Armenian Veterinary Reference Laboratory for Especially Dangerous Pathogens \\ ${ }^{3}$ Armenian Reference Laboratory for Especially Dangerous Pathogens
}

June 22, 2020

\begin{abstract}
Brucellosis remains one of the major zoonotic diseases worldwide and requires a One Health approach for early detection and control. One of the crucial components for brucellosis control and spread is timely and reliable laboratory diagnosis. External quality assessment is a key component of laboratory quality assurance to evaluate performance and identify possible insufficiencies in laboratory practices. Implementation of brucellosis external quality assessment in resource-limited countries are rare and challenging due to logistical and financial difficulties. The aim of this study was to evaluate the stability of dried tube specimens for external quality assessment of brucellosis serological testing that could be used in resource-limited countries to avoid logistical and financial constraints associated with use of sera. Prepared dried tube specimen panels consisted of 5 samples, one negative and 4 positive samples ranging from weak positive to strong positive. It was shown that brucellosis dried tube specimens were stable at room temperature for 105 days ( 15 weeks). Consistent results were observed for all samples by rose bengal test during weekly stability testing and also at the end of the stability period by complement fixation test, enzymelinked immunosorbent assays, and fluorescence polarization assay. In conclusion, brucellosis dried tube specimen maintains integrity of serum samples for serological testing of brucella infection and can be a powerful tool for external quality assessment providers, as it decreases huge shipping costs and avoids challenges in maintaining cold chain shipments between the provider and the recipient laboratories. Moreover, it has great prospects for enabling expansion of external quality assessment programs to include lower tier labs in resource-limited countries to monitor and improve the quality and accuracy of brucellosis testing, as well as it could be used for transportation of clinical samples from remote areas without cold chain logistics.
\end{abstract}

Dried tube specimen preparation and stability validation for brucellosis serological external quality assessment and quality control materials in resource-limited settings

Short running title: Brucellosis dried tube specimen validation

Arsen Zakaryan ${ }^{1,3}$, Julius Manjengwa ${ }^{1}$, Hrant Danielyan ${ }^{2}$, Pertsh Tumanyan², Zaruhi Davtyan ${ }^{1}$, Obert Kachuwaire $^{1}$, Antoine Pierson ${ }^{1}$

${ }^{1}$ Integrated Quality Laboratory Services (IQLS), Lyon, France

${ }^{2}$ Armenian Veterinary Reference Laboratory for Especially Dangerous Pathogens, Yerevan, Armenia

${ }^{3}$ Corresponding author

\section{Summary}


Brucellosis remains one of the major zoonotic diseases worldwide and requires a One Health approach for early detection and control. One of the crucial components for brucellosis control and spread is timely and reliable laboratory diagnosis. External quality assessment is a key component of laboratory quality assurance to evaluate performance and identify possible insufficiencies in laboratory practices. Implementation of brucellosis external quality assessment in resource-limited countries are rare and challenging due to logistical and financial difficulties. The aim of this study was to evaluate the stability of dried tube specimens for external quality assessment of brucellosis serological testing that could be used in resource-limited countries to avoid logistical and financial constraints associated with use of sera. Prepared dried tube specimen panels consisted of 5 samples, one negative and 4 positive samples ranging from weak positive to strong positive. It was shown that brucellosis dried tube specimens were stable at room temperature for 105 days (15 weeks). Consistent results were observed for all samples by rose bengal test during weekly stability testing and also at the end of the stability period by complement fixation test, enzyme-linked immunosorbent assays, and fluorescence polarization assay. In conclusion, brucellosis dried tube specimen maintains integrity of serum samples for serological testing of brucella infection and can be a powerful tool for external quality assessment providers, as it decreases huge shipping costs and avoids challenges in maintaining cold chain shipments between the provider and the recipient laboratories. Moreover, it has great prospects for enabling expansion of external quality assessment programs to include lower tier labs in resource-limited countries to monitor and improve the quality and accuracy of brucellosis testing, as well as it could be used for transportation of clinical samples from remote areas without cold chain logistics.

Keywords: brucellosis, dried tube specimen, external quality assessment, one health, proficiency testing, quality control

\section{Introduction}

Brucellosis is a leading zoonotic bacterial disease that is reported annually in disease-endemic regions (Khan, 2018, Johansen, 2017, Pappas, 2006). Brucellosis remains one of the major zoonotic diseases worldwide requiring a One Health approach for early detection, surveillance, control and elimination. Veterinary and human diagnostic laboratories play a key role in brucellosis surveillance and detection as definitive diagnosis is possible only based on laboratory results. Therefore, the reliability and quality of laboratory test results becomes key.

Laboratory quality assurance (QA) is a set of activities to ensure quality in the preanalytical, analytical, and post analytical processes. One of the main components of laboratory quality management is implementation in routine practice of internal quality control (IQC) and external quality assessments (EQA) sometimes referred to as proficiency testing (PT). IQC is the systematic and planned activities to monitor test performance to ensure that minimum levels of quality have been met. For a testing laboratory, this is to ensure test processes are working correctly and results are within the expected parameters and limits (WHO, 2001). EQA is the determination of a laboratory's performance by testing specimens of undisclosed content, sent by an independent organizer. Participation in proficiency testing enables the laboratory to assess and demonstrate the reliability of results by comparison with those from other participating laboratories and thus to evaluate the performance and identify possible insufficiencies in laboratory practices (OIE, 2018, Chapter 1.1.5). Numerous commercially available international EQA programs are costly for most resource-limited countries. Logistical challenges whether for EQA or for clinical samples coupled with need to maintain the cold chain during transportation are also complicating factors for these countries. Shipping internationally potentially infectious samples can also complicate export and import procedures and increase costs.

Early detection and confirmation of the infection is essential to reduce the spread of the disease for which the samples require transportation in cold chain, which is difficult to maintain in resource-limited countries, where the collection, storage and transportation of the samples at appropriate temperatures are either not developed or broadly not in practice. To overcome the challenges involved in transportation and storage of traditional liquid EQA samples or quality control materials, we propose a simple cost-effective and userfriendly dried tube specimen (DTS) approach. DTS specimens first developed for characterization of HIV proficiency test panels (Parekh, 2010) were successfully implemented in resource-limited settings (Nguyen, 
2015, Beber, 2015, Ramos, 2013) and their application continue to expand as they do not require cold chain which greatly reduces transportation and storage costs, as well as biosafety risks.

The numerous advantages of DTS over serum led to the planning of a stability study of DTS for Brucella serology testing panels that could be used in resource-limited countries and beyond. A stability evaluation of brucella DTS at room temperature over 105 days was undertaken for preparation of quality control specimens.

\section{Materials and Methods}

\section{Serum specimens}

Bovine whole blood (for serum) was obtained by collecting jugular venous blood into glass tubes during routine brucellosis surveillance in Armenia. Serum was transferred into separate tubes and tested by rose bengal test (RBT). Positive samples were confirmed by complement fixation test (CFT) and enzyme-linked immunosorbent assays (ELISA), and stored at $-20^{\circ} \mathrm{C}$. Several negative and positive sera were pooled together to produce negative and positive batches. In order to limit the risk of bacterial contamination, all the sera used were filtered through $0.22 \mu \mathrm{m}$ PVDF low protein binding filter (Millipore, Sigma) before the preparatory step. Pooled serum was tested by RBT before and after filtration.

\section{Ethics statement}

For this study no ethical approvals were required. All bovine blood samples were routinely collected for prescribed diagnostic purposes or official monitoring studies and subsequently anonymized made available for this study.

\section{Sample volume used for DTS panel}

To determine the optimal volume needed for preparing of DTS, various amounts $(30,40,50$, and $100 \mu \mathrm{L})$ of the serum were dispensed into the bottom of transparent $2 \mathrm{~mL}$ screw-cap cryogenic vials. The tubes were left open and allowed to dry at room temperature for 24 hours. The following day, samples were inspected visually for confirmation of desiccation and the tubes were capped. Rehydration of DTS samples was performed by adding distilled water and incubation at room temperature for 10-15 minutes. Then, specimens were mixed by gentle tapping without vortexing for complete solubilization of dried serum.

Liquid samples of the same pooled serum were stored at $2-8^{\circ} \mathrm{C}$ overnight to perform comparative analysis of DTS with the liquid samples.

\section{Preparation of DTS panels for stability evaluation}

The dilutions of the positive sera were carried out with negative sera. Serial dilutions were used to dilute positive sera to produce 4 levels of positive samples ranging from strong positive to weak positive, which was diluted 1:4. The negative serum samples were similarly prepared for DTS. Sufficient quantity of DTS panels were prepared for 15 weeks stability evaluation (based on the weekly testing), In addition, about $20 \%$ extra samples were prepared for any unexpected concern. All samples were stored at room temperature during the period of stability evaluation.

\section{$R B T$}

All tests were performed in duplicates. Reagents and quality control samples were allowed to reach to room temperature before use. The RBT (Biomerieux, France) was performed using a validated kit according to the manufacturer's instruction. The test was performed by thoroughly mixing $30 \mu \mathrm{L}$ of the test serum or controls with $30 \mu \mathrm{L}$ of antigen suspension on tear-drop ceramic plate, slowly rocked and incubated for 4 minutes. The presence of agglutination was observed immediately after the incubation.

\section{Complement Fixation Test}

The Brucella abortus strain 99-Weybridge (Pourquier CFT Brucellosis Ag, IDEXX, US) was used. This antigen was standardized to give $50 \%$ of fixation with a final dilution of $1 / 200$ of the International Brucella 
abortus Standard Serum (OIEISS). The CFT was performed according to standard procedures as detailed in the OIE Terrestrial Manual (OIE, 2018, Chapter 3.1.4). Briefly, serum samples were inactivated at $59^{\circ} \mathrm{C}$ for 30 minutes and diluted to cover a dilution range from 1/4 to $1 / 512$ in Veronal buffer (IDEXX, US). A total of $25 \mu \mathrm{L}$ of diluted sera, equivalent volume of antigen diluted according to the manufacturer's (IDEXX, US) instructions and $2 \mathrm{U}$ complement (IDVet, France) were added to the tubes and incubated overnight at $2-8^{\circ} \mathrm{C}$. After incubation, $25 \mu \mathrm{L}$ of $2 \%$ sheep red blood cells sensitized with rabbit anti sheep red blood cell antibodies, were added and the tubes were incubated at $37^{\circ} \mathrm{C}$ for 30 minutes, then centrifuged at $1000 \mathrm{~g}$ for 10 minutes. Positive and negative controls were included in the test. The cutoff value for the CFT was [?] $50 \%$ of hemolysis inhibition $(++)$ at the $1 / 4$ dilution, corresponding to 20 International Units (IU/mL). Serum was considered positive for antibodies to Brucella spp. if it was positive to both the RBT and the CFT.

Fluorescence Polarization Assay (FPA)

FPA was performed in duplicates using Brucella FPA test kit (Ellie LLC, USA) according to the manufacturer's instruction. Reagents were allowed to come to room temperature. The test was performed by mixing $20 \mu \mathrm{L}$ of serum, positive and negative controls with $1 \mathrm{~mL}$ of sample diluent and incubated at room temperature for 5 minutes. Blank readings of all samples and controls were taken using the FPA device (Brucella FPA ${ }^{\circledR}$, Diachemix, LLC, USA), and the millipolarization $(\mathrm{mP})$ units were recorded. This was followed by addition of $10 \mu \mathrm{L}$ of the tracer to each tube mixing gently and incubating for 3 minutes, after which a second reading was obtained. For the samples, the change in $\mathrm{mP}$ was determined by deducting the negative control $\mathrm{mP}$ from each sample $\mathrm{mP}$ (based on the mean of the duplicates). The $\Delta \mathrm{mP}$ values [?] 10 were considered as negative, between 10 to 20 were considered as suspect, and values $>20$ were considered as positive. A typical threshold level for the absolute $\mathrm{mP}$ is above $95 \mathrm{mP}$ units.

\section{ELISA}

The indirect ELISA test for the detection of antibodies againstBrucella abortus, melitensis or suis was performed according to the manufacturer (IDVet, France) instruction. The test was Calibrated according to OIE specifications and Annex C of the European directive CEE 64/432 to correctly detect the OIEELISAspISS standard serum.

\section{Results}

\section{Determination of optimal volume}

Different volumes $(30,40,50$, and $100 \mu \mathrm{L})$ of serum samples were left for approximately 24 hours at room temperature to air dry. Tubes with 30,40 , and $50 \mu \mathrm{L}$ were well dried having a yellowish pellet stuck to the bottom of the tubes. Tubes with $100 \mu \mathrm{L}$ were not completely dried. Thus, $50 \mu \mathrm{L}$ volume of bovine serum was chosen to produce DTS panels and all subsequent testing was conducted with the DTS prepared using $50 \mu \mathrm{l}$ volume of serum.

\section{Assessing the filtration and comparison of DTS with liquid specimens}

The results of serum tested before and after filtration were consistent. Similar results were obtained by RBT for all levels of DTS samples when compared with the liquid samples used to prepare DTS batch on the day of preparation $\left(\mathrm{D}_{0}\right)$. These data indicate that anti-brucella agglutinating antibodies were not affected by filtration or air drying during 24 hours at room temperature.

\section{Stability of DTS panel}

The stability of DTS samples at room temperature was performed based on weekly evaluation for 15 weeks (105 days, $\left.\mathrm{D}_{105}\right)$ using RBT, results are presented in Table 1. Additionally, on day $105\left(\mathrm{D}_{105}\right)$ testing was performed using CFT, ELISA and FPA to evaluate and confirm the stability of DTS samples, these results are presented in Table 2. The 105 days period was chosen as sufficient time for EQA organizers to produce panels, validate them, send them out and have some time until closure of EQA (usually 8 to 10 weeks is required) in case of queries. Moreover, for the laboratory IQC, the longer the samples are stable, the better 
for the laboratory. As shown in tables 1 and 2, DTS panel has a minimum stability of 105 days for testing by RBT, CFT, ELISA and FPA. These data indicate that DTS could successfully be used both for brucellosis EQA panel production and for laboratory IQC material preparation.

\section{Discussion}

Previous investigations have successfully assessed application of DTS for HIV, syphilis, and hepatitis B proficiency testing in resource-limited settings (Mendiratta, 2017, Benzaken, 2014, Ramos, 2013). Experience from many resource-limited countries shows that traditional EQA programs, when they exist, are only limited to national and sometimes regional laboratories without participation from lower tier laboratories. Our intent was to assess stability of DTS as a mean of proficiency testing panels preparation for serological testing of brucellosis to overcome the challenges related to expenses for cold chain maintenance during transportation and storage of liquid samples and biosafety concerns, as it is one of the limiting factors for developing countries.

Taking into consideration One Health approach and the challenges with international EQA in resourcelimited settings, we have launched a national EQA survey for serological tests of brucellosis in Armenia simultaneously for both human and veterinary laboratories. At the same time, we have prepared DTS to validate stability to minimize the cost and ensure long term sustainability of national EQA. The most common serological screening tests used are RBT also called buffered Brucella antigen test (OIE, 2018, Chapter 3.1.4) and serum agglutination test (SAT) which uses serum to detect the presence of agglutinating antibodies to the smooth lipopolysaccharide (S-LPS) or O-polysaccharide (OPS) as a mean of initial diagnosis of Brucella infection, particularly in lower tier laboratories. Secondary confirmatory tests such as CFT, ELISA and/or FPA are recommended as no single serological test is appropriate in all epidemiological situations because all tests have limitations, especially when screening individual animals (OIE, 2018, Chapter 3.1.4). These led us to use these techniques for DTS panel validation.

Results obtained in this study validate the long term (105 days) stability of DTS preparation for brucellosis serological testing. Therefore, the implementation of the novel approach described in this study provides a simple solution for organization of brucellosis serological EQA programs in resource-limited settings. The same strategy could also be used to prepare laboratory internal quality control specimens at national level that could be then distributed to the regional and district laboratories. Moreover, this method could also be implemented in countries experiencing challenges of storage, cold chain maintenance, and timely transport of brucellosis clinical samples from remote areas, point-of-care or lower tier laboratories to the central level for diagnosis or conformational purposes. Thus, the brucellosis DTS method has several advantages over traditional methods: 1) it is easy and cheap to prepare, 2) it is safer and less biohazardous (no spills risk during transportation), 3) the specimens could be transported and stored at room temperature without the need for maintaining expensive cold chain, and 4) as demonstrated here, to have stability over a long period of time.

In conclusion, brucellosis DTS maintain integrity of serum samples for serological testing of brucellosis for the indicated period and can be a powerful tool for EQA providers, as it decreases shipping costs and avoids challenges of maintaining cold chain shipments between the provider and recipient laboratories. Moreover, within One Health context, dried tube specimens have great prospect to enable EQA programs to reinforce veterinary and human laboratories collaboration. The utility of this tool also includes increasing coverage of EQA to the lower tier labs in resource-limited countries, with minimal costs to monitor and improve the quality and accuracy of brucellosis testing for veterinary and human laboratories.

\section{Acknowledgments}

The development of this publication was supported by the International Science and Technology Center and the United States Defense Threat Reduction Agency project implemented in Armenia. The authors are thankful to Armenian National Center for Disease Control and Prevention and all participating laboratories for helping to organize and validate the national brucella EQA program. 


\section{Conflict of Interest}

The authors declare no conflict of interest with respect to the research, authorship, and/or publication of this article.

\section{Data Availability Statements}

The authors confirm that the data supporting the findings of this study are available within the article.

\section{References}

Beber, A. M., Sabidó, M., Vieira, J, M., Bazzo, M. L., Benzaken, A. S. (2015) External quality assessment in the voluntary counseling and testing centers in the Brazilian Amazon using dried tube specimens: results of an effectiveness evaluation. Rev Soc Bras Med Trop, 48 Suppl, 1, 87-97.doi: 10.1590/0037-8682-01062014 Benzaken, A. S., Bazzo, M. L., Galban, E., Pinto, I. C., Nogueira, C. L., Golfetto, L., Benzaken, N. S., Sollis, K. A., Mabey, D., Peeling, R. W. (2014). External quality assurance with dried tube specimens (DTS) for point-of-care syphilis and HIV tests: experience in an indigenous population screening program in the Brazilian Amazon. Sex Transm Infect., 90, 14-18.doi: 10.1136/sextrans-2013-051181 Johansen, M. V., Welburn, S. C., Dorny, P., Brattig, W. N. (2017). Control of neglected zoonotic diseases. Acta Trop., 165, 1-2.doi: 10.1016/j.actatropica.2016.11.036 Khan, M. Z., \& Zahoor, M. (2018). An overview of brucellosis in cattle and humans, and its serological and molecular diagnosis in control strategies. Trop Med Infect Dis., 3, 65. doi: https://doi.org/10.3390/tropicalmed3020065 Mendiratta, S., Kamal, C. M., Sharma, S., Chhabra, Reba., Singh, S. (2017). Dried tube specimens: A tool to ensure effective proficiency testing \& quality control of Hepatitis B virus diagnosis in developing countries. International Journal of Biomedical and Advance Research, 8, 233-238. Published online: https://www.ssjournals.com/index.php/ijbar/article/view/4194/2823 Nguyen, S., Ramos, A., Chang, J., Li, B., Shanmugam, V., Boeras, D., Nkengasong, J. N., Yang, C., Ellenberger, D. (2015). Monitoring the quality of HIV-1 viral load testing through a proficiency testing program using dried tube specimens in resource-limited settings. J Clin Microbiol, 53,1129-36.doi: 10.1128/JCM.02780-14 OIE (2018). Manual of Diagnostic Tests and Vaccines for Terrestrial Animals. Chapter 1.1.5, Quality management in veterinary laboratories. ISBN 978-92-95108-18-9 OIE (2018). Manual of Diagnostic Tests and Vaccines for Terrestrial Animals. Chapter 3.1.4, Brucellosis (Brucella abortus, B. melitensis and B. suis) (infection with B. abortus, B. melitensis and B. suis). ISBN 978-92-95108-18-9 Pappas, G., Papadimitriou, P., Akritidis, N., Christou, L., Tsianos, V.T. (2006). The new global map of human brucellosis. Lancet Infect. Dis., 6, 91-99.doi: 10.1016/S1473-3099(06)70382-6 Parekh, B. S., Anyanwu, J., Patel, H., Downer, M., Kalou, M., Gichimu, C., Keipkerich, B. S., Clement, N., Omondi, M., Mayer, O., Ou, C. Y., Nkengasong, J. N. (2010). Dried tube specimens: A simple and cost-effective method for preparation of HIV proficiency testing panels and quality control materials for use in resource-limited settings. J Virol Methods, 163, 295-300.doi: 10.1016/j.jviromet.2009.10.013 Ramos, A., Nguyen, S., Garcia, A., Subbarao, S., Nkengasong, J. N., Ellenberger, D. (2013). Generation of dried tube specimen for HIV-1 viral load proficiency test panels: a cost-effective alternative for external quality assessment programs. J Virol Methods, 188,1-5.doi: 10.1016/j.jviromet.2012.11.036 WHO (2001). Laboratory quality management system: handbook. ISBN: 9789241548274

\section{Hosted file}

Table 1 Stability tets by RBT.docx available at https://authorea.com/users/335107/articles/ 461003-dried-tube-specimen-preparation-and-stability-validation-for-brucellosisserological-external-quality-assessment-and-quality-control-materials-in-resourcelimited-settings

\section{Hosted file}

Table 2 Stability test by CFT_ELISA_FPA.docx available at https://authorea.com/users/ 335107/articles/461003-dried-tube-specimen-preparation-and-stability-validation-forbrucellosis-serological-external-quality-assessment-and-quality-control-materials-inresource-limited-settings 